\title{
Asymptomatic spinal cord lesions in clinically isolated optic nerve, brain stem, and spinal cord syndromes suggestive of demyelination
}

\author{
J I O'Riordan, N A Losseff, C Phatouros, A J Thompson, I F Moseley, D G MacManus,
} W I McDonald, D H Miller

\begin{abstract}
Objectives-Conventional T2 weighted MRI studies have highlighted the fact that the presence of clinically silent brain lesions increases the risk of developing clinically definite multiple sclerosis after an isolated syndrome of the optic nerve, brain stem, or spinal cord. The objectives of the present study are: (1) to show whether or not these patients also have asymptomatic abnormalities of the spinal cord, and (2) to recruit a new cohort of such patients using high resolution MRI of both brain and spinal cord.
\end{abstract}

Methods-The brain was imaged in the axial plane with $3 \mathrm{~mm}$ thick contiguous slices using a proton density and $T 2$ weighted fast spin echo (FSE) sequence; a $T 1$ weighted sequence after the injection of gadolinium-DTPA; and a fast fluid attenuated inversion recovery (fFLAIR) sequence. The spinal cord was imaged in the sagittal plane with $3 \mathrm{~mm}$ thick slices using a T2 weighted FSE and a T1 weighted gadolinium enhanced sequence. Results-Thirty three patients, mean age 31 (16-46) were recruited. There were 14 men and 19 women. Brain MRI was abnormal in $22(67 \%)$; no patient was seen with abnormalities on only one or other sequence. Six patients $(18 \%)$ displayed one or more gadolinium enhancing lesions on brain MRI. In the spinal cord, nine (27\%) patients displayed one or more clinically silent lesions on FSE. Two patients showed one and two gadolinium enhancing lesions in the spinal cord respectively.

Conclusion-This high incidence of spinal cord lesions emphasises that asymptomatic demyelinating lesions may also involve clinically eloquent pathways. Follow up studies are required to determine their prognostic importance.

(F Neurol Neurosurg Psychiatry 1998;64:353-357)

Keywords: isolated syndrome; spinal cord

Adults presenting with a clinically isolated syndrome of the CNS which is suggestive of demyelination have a substantial risk of developing clinically definite multiple sclerosis. ${ }^{1-7}$ Some clinical studies have shown that for patients presenting with an isolated optic neuritis the overall risk of developing clinically definite multiple sclerosis is between $34 \%$ and $75 \%$. A definitive diagnosis cannot be made at presentation, however, as dissemination in time and place has not occurred. ${ }^{8}$

There have been numerous MRI related studies using $\mathrm{T} 2$ weighted brain imaging in patients with isolated optic neuritis or clinically isolated syndromes of the brain stem and spinal cord. After short term follow up of one to five years, the overall risk of developing clinically definite multiple sclerosis is much greater for those patients with an abnormal brain MRI (mean 44\% (range 23\%-93\%)) than for those with a normal scan at presentation (mean $4 \%$ (range 0\%-33\%)). ${ }^{9-20}$ Furthermore, there is a correlation between the number of lesions at presentation and the subsequent risk of progression to multiple sclerosis over two to five years. ${ }^{16} 17$

These brain MRI findings are of major importance in the counselling of individual patients with regard to their prognosis and in selecting the most appropriate candidates for inclusion into therapeutic trials aimed at delaying the evolution from an isolated syndrome to definite multiple sclerosis. ${ }^{21}$ However, there are as yet no data on spinal cord MRI findings in such cohorts. The importance of spinal cord lesions lies in their presumed greater potential to result in clinical symptoms and disability than white matter lesions, involving as they do clinically eloquent pathways (pyramidal tracts, spinothalamic tracts, and posterior columns). Cord lesions are also potentially more specific for demyelination than cerebral white matter lesions, especially in older adults. ${ }^{22}$ It is thus important to determine the frequency of asymptomatic cord lesions at presentation with a clinically isolated syndrome and to assess whether their presence and extent is predictive of the future clinical course. The present study describes the frequency of such abnormalities in a prospectively recruited cohort of patients with clinically isolated syndromes.

Materials and methods

Patients were recruited from the wards and clinics at the National Hospital for Neurology and Neurosurgery, Queen Square and from the physicians' clinic at Moorfields Eye Hospital, London, UK. Inclusion criteria were (1) a clinically isolated syndrome suggestive of demyelination, involving the optic nerve, brain stem, or spinal cord; the syndrome had to be of acute (less than 14 days to peak severity) or subacute onset (14 to 28 days to peak severity); 
Table 1 Syndrome type, age at presentation, sex, and MRI findings of each patient

\begin{tabular}{|c|c|c|c|c|c|c|c|c|}
\hline \multirow[b]{2}{*}{ Patient } & \multirow[b]{2}{*}{ Syndrome } & \multirow[b]{2}{*}{ Age } & \multirow[b]{2}{*}{ Sex } & \multicolumn{3}{|c|}{ MRI brain* } & \multicolumn{2}{|c|}{$M R I \operatorname{cord}^{\star}$} \\
\hline & & & & $T 2$ & fFLAIR & $G d$ & $T 2$ & $G d$ \\
\hline 1 & ON & 21 & $\mathrm{~F}$ & - & - & - & - & - \\
\hline 2 & ON & 28 & $M$ & - & - & - & - & - \\
\hline 3 & BS & 40 & $\mathrm{~F}$ & + & + & - & - & - \\
\hline 4 & BS & 22 & $M$ & + & + & - & - & - \\
\hline 5 & BS & 30 & $M$ & - & - & - & - & - \\
\hline 6 & $\mathrm{ON}$ & 45 & $\mathrm{~F}$ & + & + & - & + & - \\
\hline 7 & ON & 46 & $\mathrm{~F}$ & + & + & - & + & - \\
\hline 8 & $\mathrm{ON}$ & 33 & $\mathrm{~F}$ & + & + & - & + & + \\
\hline 9 & ON & 35 & $M$ & + & + & - & - & - \\
\hline 10 & BS & 33 & $\mathrm{~F}$ & + & + & - & - & - \\
\hline 11 & BS & 33 & $M$ & - & - & - & - & - \\
\hline 12 & ON & 24 & $\mathrm{~F}$ & + & + & - & + & - \\
\hline 13 & $\mathrm{ON}$ & 40 & $M$ & + & + & - & + & - \\
\hline 14 & BS & 32 & $\mathrm{~F}$ & + & + & + & + & - \\
\hline 15 & $\mathrm{ON}$ & 42 & $\mathrm{~F}$ & + & + & - & + & - \\
\hline 16 & ON & 33 & M & - & - & - & - & - \\
\hline 17 & ON & 27 & $\mathrm{~F}$ & + & + & + & + & - \\
\hline 18 & $\mathrm{ON}$ & 37 & $M$ & - & - & - & - & - \\
\hline 19 & $\mathrm{ON}$ & 36 & $M$ & + & + & + & - & - \\
\hline 20 & ON & 32 & $\mathrm{~F}$ & + & + & + & - & - \\
\hline 21 & BS & 34 & $\mathrm{~F}$ & - & - & - & - & - \\
\hline 22 & SC & 26 & $\mathrm{~F}$ & + & ND & - & - & - \\
\hline 23 & SC & 25 & $\mathrm{~F}$ & - & - & - & - & - \\
\hline 24 & SC & 33 & $M$ & + & + & + & + & + \\
\hline 25 & SC & 28 & $\mathrm{~F}$ & - & - & - & - & - \\
\hline 26 & ON & 22 & $\mathrm{~F}$ & - & - & - & - & - \\
\hline 27 & $\mathrm{ON}$ & 31 & $\mathrm{~F}$ & + & + & - & - & - \\
\hline 28 & ON & 35 & $M$ & + & + & - & - & - \\
\hline 29 & $\mathrm{ON}$ & 25 & $\mathrm{~F}$ & + & + & + & - & - \\
\hline 30 & $\mathrm{ON}$ & 16 & $M$ & + & + & - & - & - \\
\hline 31 & BS & 30 & $\mathrm{~F}$ & + & + & - & - & - \\
\hline 32 & BS & 37 & $M$ & + & + & - & - & - \\
\hline 33 & BS & 28 & $M$ & - & - & - & - & - \\
\hline Total & & & & 22 & 21 & 6 & 9 & 2 \\
\hline
\end{tabular}

$\mathrm{ON}=$ optic neuritis; $\mathrm{BS}=$ brain stem syndrome; $\mathrm{SC}=$ spinal cord syndrome; $\mathrm{M}=$ male, $\mathrm{F}=$ female ND = not done; ${ }^{\star}$ Asymptomatic abnormalities present $(+)$ or absent $(-)$.

(2) male or female patients aged 16 to 50 years; (3) the onset of the syndrome was within three months of the examination; and (4) appropriate investigations did not disclose an alternative diagnosis to that of suspected demyelination. Informed consent was obtained from all patients before inclusion into the study. All patients underwent a full neurological examination and disability was rated using Kurtzke's extended disability status scale (EDSS). ${ }^{23}$

Magnetic resonance imaging was performed on a $1.5 \mathrm{~T}$ Signa (General Electric, Milwaukee, WI, USA) scanner provided by the Multiple Sclerosis Society of Great Britain and Northern Ireland. The brain was imaged in the axial plane with $3 \mathrm{~mm}$ thick contiguous slices using a proton density and $\mathrm{T} 2$ weighted fast spin

Table 2 Number of patients with an abnormal brain and spinal cord MRI (symptomatic lesions excluded) overall and for each clinical subgroup

\begin{tabular}{lrll}
\hline & No & $\begin{array}{l}\text { (+) Brain } \\
\text { MRI }\end{array}$ & $\begin{array}{l}\text { (+) Spinal } \\
\text { cord MRI }\end{array}$ \\
\hline Optic nerve & 19 & $14(74)$ & $7(37)$ \\
Brainstem & 10 & $6(60)$ & $1(10)$ \\
Spinal cord & 4 & $2(50)$ & $1(25)$ \\
Total & 33 & $22(67)$ & $9(27)$ \\
\hline
\end{tabular}

Table 3 The mean number of brain lesions on each sequence in patients $(n=22)$ with abnormal brain MRI

\begin{tabular}{llll}
\hline Site & FSE & $f F L A I R$ & T1 Post-Gd \\
\hline Discrete & $3(0-19)$ & $4(0-19)$ & $0.1(0-1)$ \\
Subcortical & $2.6(0-2)$ & $3(0-22)$ & $0.1(0-2)$ \\
Posterior fossa & $1.4(0-6)$ & $0.4(0-2)$ & $0.1(0-1)$ \\
Periventricular & $3.4(0-14)$ & $3.8(0-18)$ & $0.1(0-1)$ \\
Total & $11(1-33)$ & $11(1-37)$ & $0.45(0-2)$ \\
\hline
\end{tabular}

echo (FSE) sequence $(\mathrm{TR}=3200 \mathrm{~ms}$, effective $\mathrm{TE}=15 / 95 \mathrm{~ms})$; a $\mathrm{T} 1$ weighted sequence ( $\mathrm{TR}=600 \mathrm{~ms}, \mathrm{TE}=14 \mathrm{~ms}$ ) after the injection of gadolinium-DTPA $(0.1 \mathrm{mmol} / \mathrm{kg})$; and a fast fluid attenuated inversion recovery (fFLAIR) sequence $(T I=2600 \mathrm{~ms}, T R=11000 \mathrm{~ms}$, effective $\mathrm{TE}=143 \mathrm{~ms}, \mathrm{ETL}=8$ ). In each case the field of view was $25 \mathrm{~cm}$, matrix $256^{2}$, and NEX 1. The spinal cord was imaged in the sagittal plane with $3 \mathrm{~mm}$ thick slices using a T2 weighted FSE (TR=2500 ms, effective $\mathrm{TE}=56 / 98 \mathrm{~ms})$ and a $\mathrm{T} 1$ weighted gadolinium enhanced sequence $(\mathrm{TR}=500, \mathrm{TE}=19)$.

The images were reported by a single observer blinded to the clinical state. They were reported as normal if there were no focal abnormalities compatible with demyelination, or, in the case of a brain stem or spinal cord syndrome, when only the symptomatic lesions were seen. Images were reported as abnormal if there were one or more asymptomatic lesions compatible with demyelination. Four sites were identified in the brain: (1) posterior fossa, (2) discrete-cerebral white matter or basal ganglia discrete from cortex or ventricles, (3) subcortical/cortical-lesions in the subcortical white matter adjacent to the cortex, or in the cortex, and (4) periventricular. Lesions were graded according to their largest diameter as small ( $<5 \mathrm{~mm}$ ), medium (5-10), large $(>10)$, or confluent. Intrinsic spinal cord lesions were identified only if areas of high signal intensity were seen on both the proton density (TE $=56$ $\mathrm{ms}$ ) and $\mathrm{T} 2$ weighted ( $\mathrm{TE}=98 \mathrm{~ms})$ images. The high signal areas were located on sagittal images as being anterior, posterior, central (a margin of normal cord is visible both anterior 
Table 4 Mean number and site of spinal cord lesions in patients $(n=9)$ with an abnormal spinal cord MRI (symptomatic lesion excluded)

\begin{tabular}{lll}
\hline Site & FSE & $G d$ \\
\hline Cervical & $1.11(0-2)$ & $0.3(0-2)$ \\
Thoracic & $0.78(0-4)$ & 0 \\
Lumbar & 0 & 0 \\
Total & $1.89(1-4)$ & $0.3(0-2)$ \\
\hline
\end{tabular}

and posterior to the lesion), or full thickness (in the anteroposterior direction). Lesion size was scored according to maximum length along the cord in the sagittal image as small $(<5 \mathrm{~mm})$, medium $(5-10 \mathrm{~mm})$, or large $(>10)$. Lesion number and load were calculated for the cervical, thoracic, and lumbar cord individually, as well as for the whole cord.

\section{Results}

Thirty three patients were recruited (table 1). Their mean age was 31 (range 16-46). There were 14 male and 19 female patients; mean EDSS (as a result of the clinically isolated syndrome) 1.95 (range 0-8). Nineteen patients presented with optic neuritis, 10 with a brain stem syndrome, and four with a spinal cord syndrome (table 2). Brain MRI was abnormal in $22(67 \%)$ patients, with one or more asymptomatic, focal lesions compatible with demyelination (table 3). For those patients with an abnormal scan, abnormalities were seen on both fast FLAIR and FSE sequences and never on a single sequence alone. A larger number of cerebral hemispheric lesions was seen with fast FLAIR than FSE; the reverse applied in the posterior cranial fossa. Six patients (18\%) displayed one or more gadolinium enhancing lesions on brain MRI.

In the spinal cord, nine $(27 \%)$ patients displayed one or more clinically silent lesions on FSE (table 4). Lesions were seen with a similar frequency in the cervical and thoracic cord; no lumbar cord lesions were found. Most lesions were $<1 \mathrm{~cm}$ in length (12 out of 17 lesions (70\%)). Two patients showed one and two gadolinium enhancing lesions in the spinal cord respectively.

\section{OPTIC NEURITIS}

Nineteen patients, eight male and 11 female, mean age 32 (range 16-46), presented with optic neuritis, unilateral in 18 and bilateral (consecutive) in one. Brain MRI was abnormal in $14(74 \%)$ whereas spinal cord abnormalities were identified in seven (37\%) (figure).

BRAIN STEM SYNDROME

Ten patients, five male and five female, mean age 29 (range 18-41) presented with a brain stem syndrome. The symptomatic lesion was seen in only four instances. Cerebral hemispheric abnormalities were present in six $(60 \%)$ and the spinal cord showed additional lesions in one $(10 \%)$.

SPINAL CORD

Four patients, one man and three women, mean age 28 (range 25-32) presented with a spinal cord syndrome, partial in two and com-

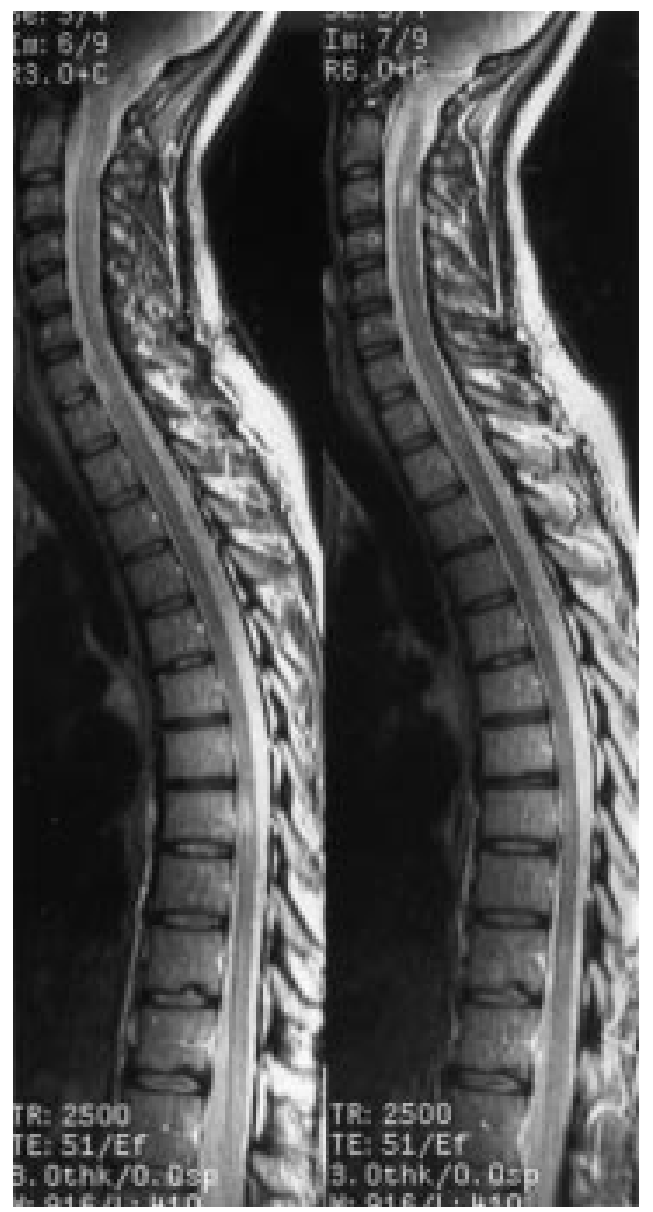

Contiguous, sagittal, $3 \mathrm{~mm}$ thick, T2 weighted images of the spinal cord in a patient with clinically isolated optic neuritis. Asymptomatic cord lesions are visible at $C 3$ and T8.

plete transverse in two The symptomatic lesion was seen in two. Brain MRI was positive in two and one showed additional abnormalities in the spinal cord.

\section{Discussion}

This study, utilising high resolution MRI and multiple imaging indices, has shown a high frequency of asymptomatic lesions not only in the brain but also in the spinal cord in patients presenting with a clinically isolated syndrome of the optic nerve, brain stem, or spinal cord. There are two particular points of interest;

Firstly, the detection of asymptomatic brain abnormalities. Previous studies, using conventional T2 weighted brain imaging in patients at presentation with a clinically isolated syndrome have generally reported multiple asymptomatic abnormalities compatible with demyelination in $50 \%-70 \%$ of patients. ${ }^{11}{ }^{1224-27}$ Our present findings using a T2 weighted FSE sequence are similar. The addition of fFLAIR did not alter the overall results - that is, no patient had abnormalities only on fFLAIR. However, consistent with previous experience, fFLAIR detected a greater number of discrete and subcortical lesions than FSE but fewer in the posterior fossa. ${ }^{28-30}$ It was also helpful in providing additional information in occasional lesions unclear on FSE. High signal on FSE due to 
demyelination may occasionally be confused with that due to vascular causes-for example, perivascular spaces or flow. Clarification of this issue is aided with the addition of fFLAIR when the last phenomena result in a low signal.

We also found six of $33(18 \%)$ patients with gadolinium enhancing lesions in the brain and only two with enhancing lesions in the spinal cord. The proportion with enhancing brain lesions is rather lower than those reported in other series and may reflect a longer interval between the onset of the syndrome and MRI. ${ }^{19-31}$ One group of investigators has reported that the risk of early progression to multiple sclerosis is higher in those with enhancing lesions than in those with nonenhancing T2 abnormalities alone. Further follow up of the present and other series is needed to clarify the longer term risks for progression to multiple sclerosis and disability. ${ }^{19}$

Secondly, the importance of asymptomatic spinal cord lesions needs to be considered. The occurrence of these abnormalities was not totally unexpected. Previous studies using somatosensory evoked potentials (SEPs) have shown that between $0 \%$ and $34 \%$ of patients with isolated optic neuritis will have some abnormality. Although all 12 patients with isolated optic neuritis studied by Hume et al had normal SEPs, Matthews reported a $10 \%$ incidence, and Paty et al a $34 \%$ incidence of abnormalities. ${ }^{32-34}$ Frederiksen et al found a greater frequency of abnormalities in SEPs taken from the tibial rather than the median nerve, presumably because of the greater length of spinal cord surveyed. ${ }^{12}$ The precise location of any abnormality was not generally reported in these studies and it may therefore have been anywhere along a path from the spinal cord to the brain stem and cerebral cortex - that is, brain lesions may result in some of the abnormalities. In those studies in which additional brain MRI was performed, there was an increase in the sensitivity of MRI over SEPs of almost $50 \% .^{12}{ }^{34}$ It is therefore clear that patients presenting in this manner may have had clinically silent episodes affecting the spinal cord as evidenced by a delay in conduction time, although their frequency is uncertain.

What is the relevance of our finding that a quarter of the patients had asymptomatic spinal cord lesions? A pathological diagnosis cannot be made with certainty as hyperintense cord lesions on T2 weighted images are non-specific, the differential diagnosis including multiple sclerosis, acute disseminated encephalomyelitis, systemic lupus erythematosus, Behçet's disease, sarcoidosis, and spinal cord infarction. ${ }^{35-39}$ Spinal cord lesions, however, are common in multiple sclerosis. In one series of 80 patients with clinically definite multiple sclerosis, $74 \%$ had intrinsic spinal cord abnormalities. ${ }^{40}$ Two patients in that study had normal brain scans but multiple lesions in the spinal cord. This is by contrast with normal controls in whom intrinsic spinal cord lesions are extremely rare. ${ }^{22}$ In particular in older patients ( $>50$ years) spinal cord lesions are more specific for demyelination than brain lesions as they do not appear with age alone in the way that cerebral white matter lesions do.

One area of concern is the possibility of acute disseminated encephalomyelitis in which spinal cord lesions may be evident. In the context of a truly monophasic illness, the presence of spinal cord abnormalities may not differentiate between acute disseminated encephalomyelitis and multiple sclerosis. In multiple sclerosis, demyelinating lesions usually affect one or two segments only (as was the case for most of the lesions we saw in this study) and may or may not be associated with cord swelling. ${ }^{20}{ }^{40}$ This contrasts with Devic's neuromyelitis optica in which the acute lesions are extensive involving many segments and with associated cord swelling. ${ }^{41}$ The MRI features of spinal cord abnormalities in acute disseminated encephalomyelitis are not as well characterised, although we have seen patients with postinfectious acute disseminated encephalomyelitis or transverse myelitis who had extensive lesions. ${ }^{42}$ That demyelinating lesions may be clinically silent even if they occur in clinically eloquent pathways is well known. Thus persistently delayed visual evoked responses are often seen after full recovery from an attack of optic neuritis, or in patients with clinically definite multiple sclerosis who have never had an attack of optic neuritis. ${ }^{43}$ Taking all the evidence together we think it likely that the cord lesions seen in our study represent areas of demyelination.

What is the role of spinal MRI in patients with clinically isolated syndromes? It is clearly important in those with isolated cord syndromes, where it has a primary diagnostic role. Although the situation is less clear in patients with isolated optic neuritis or brain stem syndromes, several potentially valuable roles emerge. Firstly, in subjects older than 50 intrinsic cord lesions are more specific for demyelination than cerebral white matter lesions, which in this age group are often due to small vessel disease. In cases in which diagnostic difficulty arises imaging of the spinal cord may help clarify the issue. Secondly, asymptomatic cord lesions might have a predictive value for the development of multiple sclerosis in the way that brain lesions do. Follow up studies are continuing on the present cohort to determine their relevance. Until this prognostic value is clarified we do not routinly image the spinal cord in all those patients with a isolated brain stem syndrome or optic neuritis. Thirdly, it would be useful to show cord lesions in those with a normal brain MRI. Although no cord lesions were found in the patients with normal brain imaging in our study, it is well documented that patients with established multiple sclerosis may have a normal brain scan but multiple cord lesions; the detection of such lesions in patients with a clinically isolated syndrome would point to a demyelinating aetiology. ${ }^{44}$ The very low frequency of gadolinium enhancing cord lesions (only one of 29 patients with optic neuritis or a brain stem syndrome) suggests a limited role for enhanced cord imaging in the diagnostic evaluation of isolated optic neuritis or brain stem 
syndromes, although we cannot exclude the possibility that more enhancing lesions would have been seen if patients had been scanned closer to the onset of their syndrome.

In conclusion, asymptomatic lesions consistent with demyelination are often seen not only in the brain but also in the spinal cord of patients presenting with a clinically isolated syndrome. Such lesions may thus be present even in clinically eloquent pathways. Whereas abnormalities in the brain confer a certain risk to the development of clinically definite multiple sclerosis and disability, it is unclear at present whether associated spinal cord abnormalities represent a similar or increased risk. Follow up studies are continuing to determine their importance.

1 Bradley WG, Whitty CW. Acute optic neuritis: prognosis for the development of multiple sclerosis. F Neurol Neurosurg Psychiatry 1968;31:10-8.

2 Compston DAS, Batchelor JR, Earl CJ, et al. Factors influencing the the risk of multiple sclerosis developing in patients with optic neuritis. Brain 1978;101:495-511.

3 Cohen M, Lessell S, Wolf P. A prospective study of the risk of developing multiple sclerosis in uncomplicated optic of developing multiple sclerosis in

4 Perkin GD, Rose FC. Optic neuritis and its differential diagnosis. Oxford: Oxford University Press, 1979.

Francis DA, Compston DAS, Batchelor JR, et al. A reassessment of the risk of multiple sclerosis developing in patients with optic neuritis after extended follow-up. $f$ Neurol Neurosurg Psychiatry 1987;50:758-765.

6 Rizzo JF, Lessell S. Risk of developing multiple sclerosis after uncomplicated optic neuritis: a long term prospective study. Neurology 1988;38:185-90.

7 Sandberg-Wolheim M, Bynke H, Cronqvist S, et al. A long term prospective study of optic neuritis: evaluation of risk factors. Ann Neurol 1990;27:386-93.

8 Poser CM, Paty D, Scheinberger L, et al. New diagnostic criteria for multiple sclerosis: guidelines for research protocols. Ann Neurol 1983;13:227-31.

9 Miller DH, Ormerod IEC, McDonald WI, et al. The early risk of multiple sclerosis after optic neuritis. $\mathcal{F} \mathrm{Neurol} \mathrm{Neu-}$ risk of multiple sclerosis after optic
rosurg Psychiatry 1988;51:1569-71.

10 Miller DH, Ormerod EC, Rudge P, et al. The early risk of multiple sclerosis following isolated acute syndromes of the multiple sclerosis following isolated acute syndromes of

11 Martinelli V, Comi MV, Filippi M, et al. Paraclinical tests in acute onset optic neuritis: basal data and results of a shor follow up. Acta Neurol Scand 1991;84:231-6.

12 Frederiksen JL, Larsson HBW, Olesen J, et al. MRI, VEP, SEP and biothesiometry suggest monosymptomatic acute optic neuritis to be a first manifestation of multiple sclerosis. Acta Neurol Scand 1991;83:343-50

13 Lee KH, Hashimoto SA, Hooge JP, et al. Magnetic resonance imaging of the head in the diagnosis of multiple sclerosis: a prospective 2 year follow-up with comparison of clinical evaluation, evoked potentials, oligoclonal banding, and CT. Neurology 1991;41:657-60.

14 Jacobs L, FE Munschauer, Kaba SE. Clinical and magnetic resonance imaging in optic neuritis. Neurology 1991;41:15resona.

15 Ford B, Tampieri D, Francis G. Long term follow up of acute transverse partial myelopathy. Neurology 1992;42 250-2.

16 Morrissey SP, Miller DH, Kendall BE, et al. The significance of brain magnetic resonance abnormalities at presentation with clinically isolated syndromes suggestive of multiple sclerosis. Brain 1993;116:135-46.

17 Beck RW, Cleary PA, Trobe JD, et al. The effect of corticosteroids for acute optic neuritis on the subsequent development of multiple sclerosis. N Engl F Med 1993;329:1764-9.

18 Filippi M, Horsfield MA, Morrissey SP, et al. Quantitative brain MRI lesion load predicts the course of clinically isolated syndromes suggestive of multiple sclerosis. Neurology 1994:44:635-41.
19 Tas MW, Barkhof F, van Walderveen MAA, et al. The effect of gadolinium on the sensitivity and specificity of MR in the initial diagnosis of multiple sclerosis. Am 7 Neuroradiol 1995;16:259-64.

20 Campi A, Filippi M, Comi G, et al. Acute transverse myelopathy: spinal and cranial MR study with clinical follow-up. Am f Neuroradiol 1995;16:115-23.

21 Miller DH, Albert PS, Barkhof F, et al. Guidelines for the use of magnetic resonance techniques in monitoring the

2 Thorpe JW, Kidd D, Kendall BE, et al. Spinal cord MRI using multi-array coils and fast spin echo. I. Technical aspects and findings in healthy adults. Neurology 1993;43: $2625-31$.

23 Kurtzke JF, Rating neurological impairment in multiple clerosis: an expanded disability status scale (EDSS). Neurology 1983;33:1444-52.

24 Jacobs L, Kinkel PR, Kinkel WR. Silent brain lesions in patients with isolated optic neuritis. A clinical and nuclear magnetic resonance imagiung study. Arch Neurol 1986;43: $452-5$.

25 Ormerod IEC, McDonald WI, du Boulay EPGH, et al. Disseminated lesions at presentation in patients with optic neuritis. $\mathcal{F}$ Neurol Neurosurg Psychiatry 1986;49:124-7.

26 Ormerod IEC, Bronstein A, Rudge P, et al. Magnetic resonance imaging in clinically isolated lesions of the brain stem. F Neurol Neurosurg Psychiatry 1986;49:737-43.

27 Miller DH, McDonald WI, Blumhardt LD, et al. Magnetic resonance imaging in isolated noncompressive spinal cord syndromes. Ann Neurol 1987;22:714-23.

28 Filippi M, Yousry T, Baratti C, et al. Quantitative assessment of MRI lesion load in multiple sclerosis; a comparison of conventional spin echo with fast fluid attenuated inversion recovery. Brain 1996;119:1349-55.

29 Stevenson VL, Gawne-Cain ML, Barker GJ, et al. Imaging of the spinal cord and brain in multiple sclerosis: a comparative study between fast flair and fast spin echo. $f$ Neurol 1997;244:119-24.

30 Gawne-Cain ML, O'Riordan JI, Thompson AJ, et al. Multiple sclerosis lesion detection in the brain: a comparison of fast fluid attenuated inversion recovery and conventional T2 weighted dual spin echo. Neurology 1997;49:364-70.

31 Christiansen P, Frederiksen JL, Henriksen O, et al. Gd-DTPA-enhanced lesions in the brain of patients with acute optic neuritis. Acta Neurol Scand 1992;85:141-6.

32 Hume AL, Waxman SG. Evoked potentials in suspected multiple sclerosis: diagnostic value and prediction of clinical course. F Neurol Sci 1988;83:191-210

33 Matthews WB. Somatosensory evoked potentials in retrobulbar neuritis. Lancet 1978;ii:443.

34 Paty DW, Oger JJF, Kastrukoff LF, et al. MRI in the diagnosis of MS. A prospective study with comparison of clinical evaluation, evoked potentials, oligoclonal banding, and CT. Neurology 1988;38:180-5.

35 Kesselring J, Miller DH, Robb SA, et al. Acute disseminated encephalomyelitis: MRI findings and the distinction from multiple sclerosis. Brain 1990;113:291-302.

36 Miller DH, Buchanan N, Barker G, et al. Gadolinium enhanced magnetic resonance imaging of the central nervous system in systemic lupus erythematosus. $f$ Neurol 1992:239:460-4.

37 Morrissey SP, Miller DH, Hermanszewski R, et al. Magnetic resonance imaging of the central nervous system in Behçets disease. Eur Neurol 1993;33:287-93.

38 Nesbit GM, Miller GM, Baker HJ, et al. Spinal cord sarcoidosis: a new finding at MR imaging with Gd-DTPA enhancement. Radiology 1989;173:839-43.

39 Yuh WTC, Marsh EE, Wang AK, et al. MR imaging of spinal cord and vertebral body infarction. Am $\mathcal{F}$ Neuroradiol 1992;13:145-54.

40 Kidd D, Thorpe JW, Thompson AJ, et al. Spinal cord MRI using multi-array coils and fast spin echo.II.Findings in multiple sclerosis. Neurology 1993;43:2632-7.

41 O'Riordan JI, Gallagher HL, Thompson AJ, et al. The clinical, cerebrospinal fluid and MRI findings in Devic's neuromyelitis optica. $f$ Neurol Neurosurg Psychiatry 1996;60:382-

42 Francis DA, Brown A, Miller DH, et al. MRI appearances of the CNS manifestations of mycoplasma pneumonia: a report of two cases. F Neurol 1988;235:441-3.

43 Halliday AM, McDonald WI, Mushin J. Delayed visual evoked response in optic neuritis. Lancet 1972;i:982-5.

44 Thorpe JW, Kidd D, Moseley IF, et al. Spinal MRI in patients with suspected multiple sclerosis and negative brain MRI. Brain 1996;119:709-14. 